
April 1939

\title{
A POTENTIOMETER FOR MEASURING VOLTAGES OF 10 MICROVOLTS TO AN ACCURACY OF 0.01 MICROVOLT
}

\author{
By Ray P. Teele and Shuford Schuhmann
}

\section{ABSTRACT}

In connection with the development of a physical photometer it was found necessary to measure voltages of the magnitude of 10 microvolts to an accuracy of 1 part in a thousand. The design and construction of a potentiometer of the desired range and accuracy are described.

\section{CONTENTS}

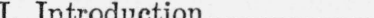

II. Potentiometer circuit

III. Thermal shield _._.

IV. Causes of extraneous thermal electromotive forces in the measurement circuit._._.

V. Reduction or elimination of extraneous thermal electromotive forces _. 433

1. Temperature differences in the space within the thermal shield_. 433

2. Temperature differences between the springs and contacts of the reversing key

3. Temperature differences between terminal leads of the fourterminal resistor

4. Temperature differences caused by heat conducted into the

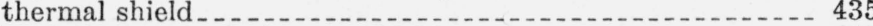

(a) Along wires entering the shield

(b) Along the actuating mechanism of the key ........ 435

VI. Other sources of extraneous electromotive forces and their reduction or elimination

1. In the unknown-voltage circuit

2 . In the galvanometer circuit.

VII. Adjustment of the reversing key VIII. Tests and performance.

\section{INTRODUCTION}

The response of a thermopile used in a physical photometer being developed at the Bureau was found to be of the magnitude of 10 microvolts. It was desired to measure voltages of this magnitude to an accuracy of 1 part in a thousand. A potentiometer of the desired range and accuracy was designed and constructed.

When a small electromotive force of this magnitude is to be measured the most serious difficulty lies in the presence of extraneous thermal electromotive forces in the various parts of the measurement circuit. The major part of the apparatus to be described is designed to prevent or eliminate the effects of such electromotive forces. 


\section{POTENTIOMETER CIRCUIT}

A brief description of the potentiometer circuit and the method of using the potentiometer will suffice for a general understanding of the equipment. The special thermal shield, the reversing key, and the means employed to prevent or eliminate the effects of extraneous electromotive forces will then be discussed.

The electrical circuit ${ }^{1}$ is shown in figure 1. In this figure, $e_{x}$ represents the unknown voltage to be measured; $A$, the thermal shield enclosing the "measurement circuit," consisting of the reversing

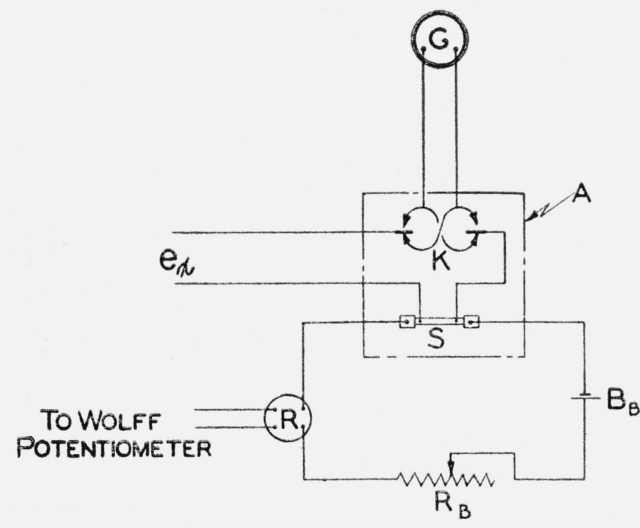

FiguRe 1.-Electrical circuit. key and the four-terminal resistor, $S$; $G$, the galvanometer; $R_{B}$, the balancing rheostat; $R$, a four-terminal resistance standard. With $e_{x}$ applied to the potentiometer, the resistance of $R_{B}$ is varied until the deflection of the galvanometer shows no change when the key, $K$, is operated. This condition indicates that the potential drop across the four-terminal resistor, $S$, which is due to the current in the balancing: circuit, is equal and opposite to $e_{x}$. The current in the balancing circuit flows through

$R$, and the potential drop across $R$ is measured by a suitable auxiliary potentiometer, in the present case a Wolff potentiometer of the Feussner type.

The reading of the Wolff potentiometer depends upon the values of $R$ and $S$. If $e_{x}$ is assumed to be 10 microvolts, or $10^{-5}$ volt, and $S$ is $0.01 \mathrm{ohm}$, the current, at balance, will be $10^{-3}$ ampere. This current flows through the $100-\mathrm{ohm}$ resistance standard $R$. The potential drop across $R$ is, therefore, $10^{-1}$ volt, which is of a satisfactory magnitude for measurement. With the particular values of $S$ and $R$ chosen here, the balancing rheostat, $R_{B}$, needs to be adjustable from 0 to $10,000 \mathrm{ohms}$ to cover a range of 2 to 2,000 microvolts (approximately) when a single lead storage cell is used to supply the balancing current. In addition to making the balancing battery and resistor of convenient size, these values for $S$ and $R$ mean that the first two dials of the auxiliary Wolff potentiometer indicate the whole microvolts, and the remaining three dials indicate the decimal parts of a microvolt.

When a lower accuracy is sufficient, a suitable milliammeter may be used to measure the current in the balancing circuit.

\section{THERMAL SHIELD}

It is desirable to reduce parasitic electromotive forces to a value of 0.002 microvolt, that is, one-fifth of the desired accuracy. To accomplish this a number of expedients are employed, one of which is the isolation of the measurement circuit inside a thermal shield.

1 J. C. Poggendorff, Ann Phys. Chem. 54, 161 (1841); Lindeck and Rothe, Z. Instrumentenk 20, 293 (1900); H. B. Brooks and A. W. Spinks, BS J. Research 9, 781 (1932). RP508. This last paper contains a brief summary of the two references above and a discussion of basic potentiometer circuits. 
The thermal shield is cast from an alloy of aluminum containing 15 percent of zinc. The zinc improves the machinability of the casting without reducing its thermal conductivity sufficiently to offset the gain in working qualities. The wall thickness is $3 / 3$ inch.

The thermal shield is rectangular in shape and the division into halves is made along a diagonal plane. Figure 2 shows the thermal shield before final assembly. The division along a diagonal plane permits easy access to the parts when one-half of the shield is removed and also has the obvious advantage of requiring only one pattern for casting.

\section{CAUSES OF EXTRANEOUS THERMAL ELECTROMO- TIVE FORCES IN THE MEASUREMENT CIRCUIT}

Thermal electromotive forces may arise from (1) temperature differences in the space within the thermal shield, (2) temperature differences between the springs and contacts of the reversing key, (3) temperature differences between the terminals of the four-terminal resistor, $S,(4)$ heat conducted into the space within the thermal shield along the wires entering the thermal shield and along the actuating mechanism of the reversing key.

\section{REDUCTION OR ELIMINATION OF EXTRANEOUS THERMAL ELECTROMOTIVE FORCES}

The various expedients used to reduce or eliminate the effects of the extraneous thermal electromotive forces are discussed according to the principal causes listed above. The various expedients used are each effective and the over-all result obtained is the product of the separate effectivenesses of the expedients used. The aim was to ensure that the precautions taken would be sufficient, and the necessity for each expedient was not determined. It is probable that an extensive study of the apparatus would show that simplification could be made by modifying or omitting some of the expedients used.

\section{TEMPERATURE DIFFERENCES IN THE SPACE WITHIN THE THERMAL SHIELD}

The perfect thermal shield would be a cavity in a block of metal of high thermal conductivity, with no openings of any kind, and thermally insulated from its surroundings. The necessity for providing for the entrance of wires and means for manipulating the reversing key, as well as the difficulties encountered in making such a thermal shield, make the ideal shield unattainable. The nearest approach to such a shield is an enclosure that is symmetrically divided. The symmetrical division of the enclosure gives two pieces of metal of very nearly equal thermal capacity. When these two halves are fastened together in such a manner that there is good thermal contact between halves, the enclosure has a symmetry of thermal capacity that greatly reduces the possibility of unequal distribution of temperature on the walls of the enclosure.

The thickness of the walls, the thermal capacity due to the mass, the thermal conductivity of the metal, and the thermal insulation are depended upon to maintain the walls of the thermal shield at the same 
temperature throughout. When the walls of the thermal shield are at the same temperature throughout the space within the shield is free from temperature gradient in any direction and, consequently, apparatus located within the thermal shield is free from temperature differences which might cause thermal electromotive forces between component parts.

The thermal shield is enclosed in a box of balsa wood 2 inches thick to minimize the flow of heat to or from the surroundings. Such a heat flow might cause one part of the thermal shield to reach a temperature differing from that of some other part.

\section{TEMPERATURE DIFFERENCES BETWEEN THE SPRINGS AND CONTACTS OF THE REVERSING KEY}

The springs of the reversing key are of copper strip, which was coldrolled to improve its elastic properties. The silver contact points are riveted in place. In order to minimize differences in temperature between any two silver-to-copper junctions which are in the measuring circuits, the fixed ends of all six springs were clamped between heavy copper blocks, with thin mica sheet for electrical insulation, and these blocks were in turn well connected mechanically and thermally to the side of the thermal shield. If, in spite of the precautions taken, the temperature of this portion of the shield should differ, however slightly from that of the rest of the shield, the symmetry of thermal capacity and thermal resistance of the six springs would still serve to maintain their free ends at the same temperature. The use of a small mica-insulated copper block to form a mechanical connection between the free ends of the inner or moving pair of springs provides for further equalization of temperature between these two springs.

In addition, the brass pieces, $B-B$ in figure 3 , make a partial enclosure within the main space, which gives still greater assurance that all parts of the reversing key are at the same temperature.

The reversing key is designed so that all junctions of dissimilar metals occur in adjacent pairs so arranged that a flow of heat in any direction will cause thermal electromotive forces of opposite sign and nearly equal magnitude in the circuit containing the key.

\section{TEMPERATURE DIFFERENCES BETWEEN TERMINAL LEADS OF THE FOUR-TERMINAL RESISTOR}

The resistor, $S$, is of the four-terminal type and is made in the form of a $U$. The open end is clamped, with mica, between copper blocks, and the copper blocks are bolted to the shield. The terminal leads are bent toward each other and brought as close together as possible without introducing any danger of a short-circuit between them, when air is the insulating medium. This brings the four terminal leads together into the same thermal environment. With the precautions taken in the design of the shield, this additional detail of grouping the terminal leads is felt to be adequate for eliminating any trouble from thermal electromotive forces at the terminals of the resistor. 


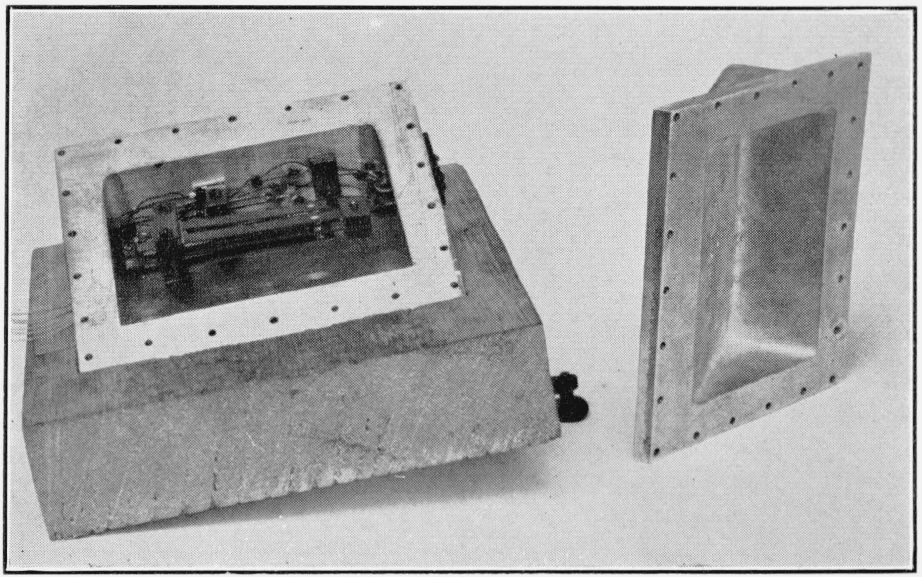

Figure 2.-Thermal shield opened.

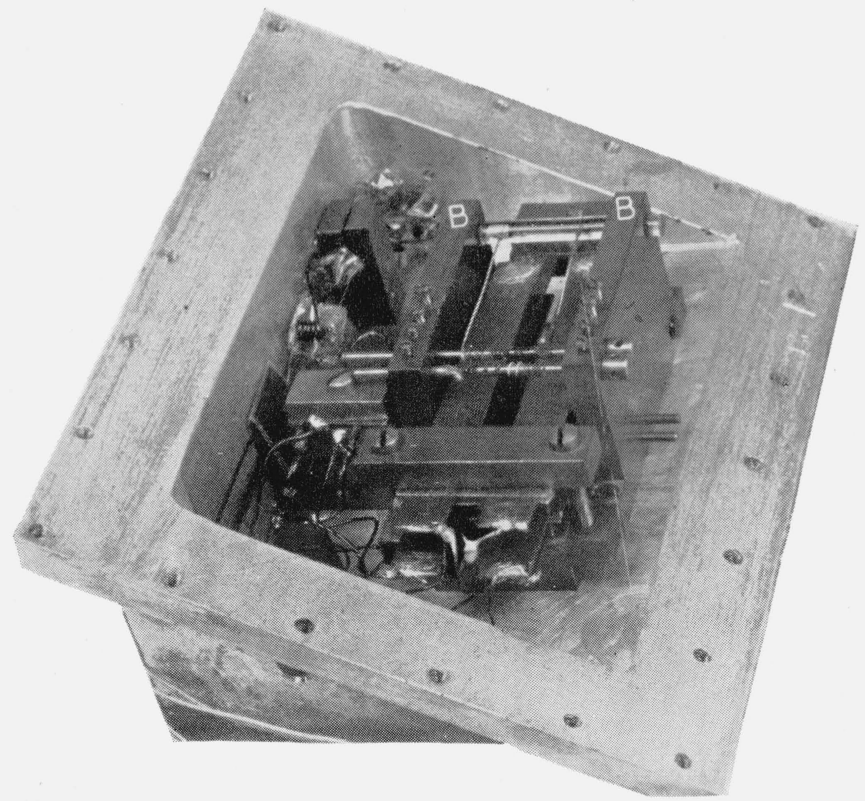

FIGURE 3.-Interior of thermal shield with mechanical stops in place. 


\section{TEMPERATURE DIFFERENCES CAUSED BY HEAT CONDUCTED INTO THE THERMAL SHIELD}

(a) ALONG WIRES ENTERING THE SHIELD

The principle of the thermal "tie-down" ${ }^{2}$ was used to divert any heat conducted along the wires onto the walls of the thermal shield. The wires enter the thermal shield through a copper block screwed to the outside of the shield. The wires are sealed with polyethylene phthalate resin, an electrical insulator but fairly good thermal conductor. About 95 percent of any heat conducted along the wires will flow to the walls of the thermal shield at this point. Just inside the shield each wire is coiled into a small helix. This helix offers a resistance to the flow of heat which increases the effectiveness of the transfer of heat at the copper block on the outside of the shield. The wires are then clamped, with mica, between copper blocks and the inner wall of the thermal shield. The heat transfer at this point is again about 95 percent complete. One more set of coils and another clamp are used before the wires are led to the various terminals. The transfer of heat from the wires to the walls of the thermal shield by these three thermal "tie-downs" is nearly complete, only 1 part in ten thousand is not diverted to the walls of the shield. In addition, the wires are led half way around the case in close proximity to the walls and to each other. Consequently, the end of each wire, where the soldered junction is made, must be at a temperature very close to that of the thermal shield and the space within the thermal shield.

(b) ALONG THE ACTUATING MECHANISM OF THE KEY

A key-actuating mechanism of usual construction would conduct heat at an undesirable rate from the hand of the operator into the thermal shield. To minimize tne rate of transfer of heat, the reversing switch is operated by a linen thread, having a metal link where it passes through the case. The use of a thread is feasible, because, normally, the key remains closed in one direction, thus furnishing its own restoring force. When a lever made of hard rubber is depressed by the operator, the opposite end of the lever pulls the linen thread. Heat conducted from the operator's hand must travel along the hardrubber lever and down the linen thread to the metal link, a path of comparatively high thermal resistance. The metal link causes most of the heat being transferred along the linen thread to flow to the walls of the thermal shield. The lower end of the metal link is attached to the movable springs by a linen thread. The hand of the operator is separated from the thermal shield by the balsa-wood enclosure.

\section{OTHER SOURCES OF EXTRANEOUS ELECTROMOTIVE FORCES AND THEIR REDUCTION OR ELIMINATION}

\section{IN THE UNKNOWN-VOLTAGE CIRCUIT}

There are several precautions that need to be observed in using the potentiometer. Obviously, the potentiometer will measure as a part of $e_{x}$ the resultant of any thermal electromotive forces in the circuit that connects this voltage to the potentiometer. To reduce these thermal electromotive forces as much as possible, any connections

2 N. S. Osborne, H. F. Stimson, and E. F. Fiock, BS J. Research 5, 425 (1930) RP209. 
should be protected from inequalities of temperature. A very satisfactory form of connector, shown in figure 4, was worked out for making these connections. Two copper bars with a copper screw in each end are clamped between metal blocks, with mica for electrical insulation. This reduces the temperature difference which can exist between the wires thus connected. The complete connector is thermally insulated and enclosed so as to exclude air currents from the junctions.

The connections must be allowed to come to thermal equilibrium with their surroundings before measurements can be made. It is apparent that connections can not be readily shifted. Consequently, when the polarity of the voltage to be measured is not known some means of reversing the potentiometer without disturbing the connections from the unknown voltage to the potentiometer is desirable.

Two reversing switches, one for reversing the current from the balancing battery, $B_{B}$ in figure 1 , and the other for reversing the poten-

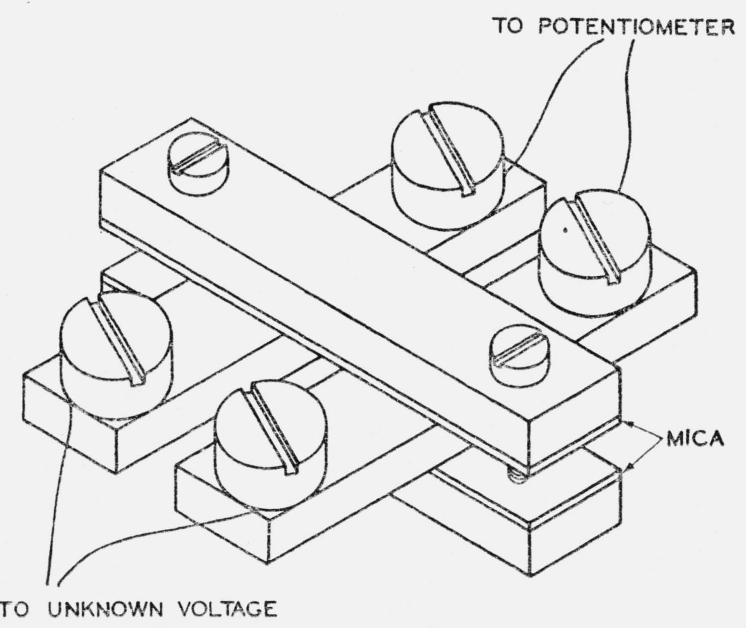

Figure 4.-Connector for leads of unknown voltage circuit.

tial leads from the standard resistor, $R$ in figure 1 , will enable the potentiometer to be balanced for either polarity of the unknown voltage. Both of these reversing switches are in the balancing circuit, where their operation will not interfere with the immediate use of the potentiometer, since the extraneous electromotive forces thus generated are in series either with the relatively large electromotive force of the battery or with the relatively large potential drop across the 100 -ohm resistor, $R$.

\section{IN THE GALVANOMETER CIRCUIT}

It is not practicable to maintain the galvanometer and galvanometer leads at a uniform temperature throughout. Consequently, there is almost always a resultant thermal electromotive force having its origin in that part of the galvanometer circuit beyond the reversing key. Under favorable conditions, for galvanometers of ordinary construction, this thermal electromotive force usually lies in the range from +1 to -1 microvolt. With an "all-copper-circuit" galvanometer 
and taking exceptional precautions it usually lies in the range from +0.1 to -0.1 microvolt. Various procedures have been devised for measuring this electromotive force so that it may be added to or subtracted from the potential drop being measured. Also, various procedures have been devised for compensating for this electromotive force ${ }^{3}$ In addition to any thermal electromotive forces in the galvanometer or galvanometer leads, there may be other extraneous electromotive forces in this portion of the circuit, for example, those produced by leakage currents from other electrical circuits.

In the present potentiometer the manner in which the reversing key is used automatically eliminates the effects of extraneous electromotive forces having their origin in the galvanometer, and in effect doubles the sensitivity of the galvanometer to a lack of balance of the potentiometer, without changing its sensitivity to either electrical or mechanical disturbing factors. The function of the reversing key has been described in detail in the preceding paper. ${ }^{4}$ For the convenience of the reader, a brief nontechnical discussion is given here. Figure 5 shows the portion of the circuit in which an extraneous electromotive force (if not taken into account) would introduce an error in the measurement.

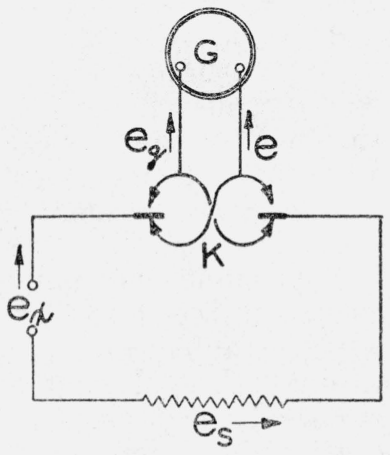

Figure 5.-Portion of electric circuit.

Let $e=$ the resultant of all extraneous electromotive forces in the galvanometer circuit. The quantity $e$ is considered as including any action upon that part of the galvanometer circuit beyond the reversing key which has any effect upon the scale readings, such as changes in the potential difference between the coil and case of the galvanometer, changes in the level of the galvanometer, and gradual shifts of the mechanical zero.

$e_{g}=$ the "unbalanced voltage," that is, the voltage due to any difference between $e_{x}$ and $e_{s}$.

$e_{x}$ and $e_{s}=$ the unknown voltage and the drop across the four-terminal resistor $S$, respectively.

Assuming exact equality of $e_{x}$ and $e_{s}$, we see that when the galvanometer reading does not change when the key, $K$, is operated it indicates (1) that $e_{\rho}$ and $e$ are both zero or (2) that $e_{j}$ is zero and $e$ is constant. As already pointed out on page $436, e$ is probably not equal to zero and we usually have the second condition.

When $e_{0}$ is not zero and the key makes contact in one position, there is a deflection in one direction, and when the key is operated we have a similar deflection in the opposite direction. This is the equivalent of doubling the sensitivity of the galvanometer to any unbalanced voltage in the measurement circuit.

'H. B. Brooks and A. W. Spinks, BS J. Research 9, 781 (1932) RP506.

$4 \mathrm{~F}$. Wenner, $A$ method of reducing the effect of disturbances in the galvanometer branch of a potentiometer cir. cuit, J. Research NBS 22, 425 (1939) RP1194. 


\section{ADJUSTMENT OF THE REVERSING KEY}

The two pairs of stationary springs are held in position by stops which may be precisely adjusted in order to obtain the necessary accurate timing of the sequence of make and break contacts which constitute a reversal. Since the springs have a position of rest where all their free ends make contact, adjustment is made by varying the length of wires connected between capstans and small clips attached to the free ends of the stationary springs, that is, the free ends of the springs are pulled from their position of rest by the wires. These wires are insulated electrically but not thermally from their metal supports.

The optimum adjustment would be such that one pair of outer blades would make contact at the exact instant the other was disengaged by the moving pair. Since the system is mechanical, this adjustment is never perfectly made, so it becomes necessary to choose whether the transient condition shall be a short-circuit or an opencircuit.

This choice is made considering the magnitude of the measured electromotive force and the characteristics of the galvanometer, with the object of causing the least momentary change of the current through the galvanometer (assuming balance for the measuring circuit). In any case the adjustment is made as near as possible to optimum and in operation the key is depressed quickly, in order that the time interval between steady states of the current may be very short.

\section{TESTS AND PERFORMANCE}

In order to test the effectiveness of the measures employed to eliminate the effects of extraneous electromotive forces in the vital parts of the circuit and to learn the operational characteristics, the reversing key and four-terminal resistor, enclosed in the thermal shield, were set up as for use, employing a high-sensitivity galvanometer.

A small all-copper shunt, well-insulated thermally, and with its potential terminals symmetrically placed and insulated from each other electrically but not thermally, was connected at leads $e_{x}$, figure 5. A known small current flowed through this shunt, which served to determine the galvanometer sensitivity without introducing any spurious thermal electromotive force.

The sensitivity of the galvanometer was found to be about $50 \mathrm{~mm}$ (double deflection) per microvolt. Upon removing the current connections from the shunt (leaving only thermal electromotive forces), it was found that on days when there was freedom from wind and magnetic disturbances, operating the key produced no discernible change in the galvanometer index reading. (The index could ordinarily be read to about $0.2 \mathrm{~mm}$ and changes of $0.1 \mathrm{~mm}$ were detectable.) This test indicated that thermal electromotive forces in the measuring circuit were certainly less than 0.002 microvolt.

The effect of a relatively large thermal electromotive force in the galvanometer circuit was also investigated. The all-copper shunt was used to supply 1 microvolt in the galvanometer circuit and the same procedure as above was followed except that the copper $e_{x}$ leads were short-circuited. Although the index reading was altered from 
that obtained in the first test, again there was no noticeable change upon reversal.

Finally, a coil of silk-covered copper wire, thermally insulated, was connected at $e_{x}$ (fig. 5), leaving the potential difference of a microvolt in the galvanometer circuit. The resistance of this coil was equal to the external critical-damping resistance (about $60 \mathrm{ohms}$ ) of the galvanometer. In this case the operation of the reversing key produced a noticeable change in index reading, which appeared to be a result of a small residual thermal electromotive force in the damping resistor and not ascribable to an impulse caused by the operation of the key.

From the above tests it can be seen that with this apparatus it is possible to measure potential differences of the order of 1 microvolt to at least 0.2 percent, even though the galvanometer circuit itself contains an electromotive force of a microvolt. However, as pointed out above, it is advisable to keep the latter electromotive force small so as to minimize any impulse caused by the operation of the key. When this electromotive force is small its variation is also small.

The authors are indebted to many members of the staff of the National Bureau of Standards for suggestions and advice. Dr. Wenner suggested the use of the reversing key and reviewed the design before construction started.

Washington, January 20, 1939. 